University of Wollongong

Research Online

Faculty of Informatics - Papers (Archive)

Faculty of Engineering and Information

Sciences

$1-1-2007$

\title{
The acceptance of a clinical IT innovation by the care givers in residential aged care 11-weeks after the software implementation in Australia
}

Ping Yu

University of Wollongong, ping@uow.edu.au

Hui Yu

University of Wollongong

Yi Mu

University of Wollongong, ymu@uow.edu.au

Follow this and additional works at: https://ro.uow.edu.au/infopapers

Part of the Physical Sciences and Mathematics Commons

\section{Recommended Citation}

Yu, Ping; Yu, Hui; and Mu, Yi: The acceptance of a clinical IT innovation by the care givers in residential aged care 11-weeks after the software implementation in Australia 2007, 6549-6552.

https://ro.uow.edu.au/infopapers/2236

Research Online is the open access institutional repository for the University of Wollongong. For further information contact the UOW Library: research-pubs@uow.edu.au 


\title{
The acceptance of a clinical IT innovation by the care givers in residential aged care 11-weeks after the software implementation in Australia
}

\author{
Abstract \\ End user acceptance and satisfaction with a new IT innovation is the pre-requisite for the successful \\ introduction of this IT solutino into an organization. More than 70 per cent of health IT projects have failed \\ to a certain extent because of its failure to satisfy the functional or non-functional requirements of the \\ end users and thus were not accepted by them. To date, there is no sound evidence to suggest that \\ clinical IT solutions will bring in benefits for a residential aged care facility. This is a real concern for aged \\ care management in investment in clinical IT solutions in Australia. Therefore, successful cases about the \\ acceptance and benefits of the introduced clinical IT solutions by care givers in aged care services are \\ essential for supporting the IT investment decisions of the aged care management and government \\ policy makers. This is an essential first step towards the diffusion of clinical IT solutions into aged care \\ sector.

\section{Keywords} \\ aged, residential, innovation, acceptance, implementation, after, weeks, 11, care, clinical, australia, \\ software, givers

\section{Disciplines} \\ Physical Sciences and Mathematics

\section{Publication Details} \\ Yu, P., Yu, H. \& Mu, Y. (2007). The acceptance of a clinical IT innovation by the care givers in residential \\ aged care 11-weeks after the software implementation in Australia. International Conference on Wireless \\ Communications, Networking and Mobile Computing (pp. 6549-6552). Piscataway, NJ: IEEE.
}




\title{
The acceptance of a clinical IT innovation by the care givers in residential aged care 11-weeks after the software implementation in Australia
}

\author{
Ping Yu, Hui Yu, Yi Mu
}

\begin{abstract}
End user acceptance and satisfaction with a new IT innovation is the pre-requisite for the successful introduction of this IT solutino into an organization. More than 70 per cent of health IT projects have failed to a certain extent because of its failure to satisfy the functional or non-functional requirements of the end users and thus were not accepted by them. To date, there is no sound evidence to suggest that clinical IT solutions will bring in benefits for a residential aged care facility. This is a real concern for aged care management in investment in clinical IT solutions in Australia. Therefore, successful cases about the acceptance and benefits of the introduced clinical IT solutions by care givers in aged care services are essential for supporting the IT investment decisions of the aged care management and government policy makers. This is an essential first step towards the diffusion of clinical IT solutions into aged care sector.
\end{abstract}

Keywords: health information system, user acceptance, residential aged care, aged care, health

\section{Background}

Nursing documentation is central to the successful operation of an aged care facility, to quality nursing care and to the appropriate and accurate management of people needing the care. It is at the heart of a residential aged care facility's comprehensive analytical communication system between and among the different categories and levels of carers. Consequently, the process of documentation forms part of the overall organisational processes in residential aged care [1].

Paper-based nursing documentation practice, however, is beset by a number of well-recognised difficulties, particularly the high documentation effort and poor quality $[2,3,4,5]$. Information technology (IT) is seen by many as the solution to these problems $[6,7,8,9,10]$. However, not much evidence is available in regard with what works and what does not work in the implementation of IT solutions in residential aged care. Lack of evidence of user acceptance and usage of the system and a perceived possible resistance to change of documentation practice are concerns for the management in making decisions about investment in clinical IT solutions.
Therefore, this article is focused on reporting the software functions that the care givers like or dislike; the documentation time, which is of management concern and the care givers' acceptance of the introduced system. The importance of end user training and support are also identified.

\section{Methodology}

This research took a case study approach. The research findings were drawn from qualitative and quantitative investigation from two aged care homes, one implemented electronic nursing documentation system and the other paper-based. Both homes belong to the same aged care management group $R S L$ Care. It examined survey data collected at RSL Care Tantula Rise, and at Centaur Memorial Retirement Community (RSL Care Centaur). The field survey period was September 29 to November 8, 2006.

To ensure that the respondents' perceptions about their facility's documentation system were comparable, the demographic profile, organisational culture and subjective norm for the use of the relevant documentation system in the two nursing homes were compared. Carers' satisfaction with the nursing documentation system that they used (including, in the case of RSL Care Tantula Rise, its hardware support as well as the information made available by the system), their perceived organisational and personal benefits from using the documentation system and their perceptions about support and training for using the system were also measured. Finally the care workers' attitude towards using electronic information system was measured.

Interview discussions were audio-taped, transcribed into a Word document through audio dictation using software Dragon Naturally Speaking Professional v8.0. Concepts presented in the discussion were extracted using NVIVO software (rf: http://www.qsrinternational.com) for every transcript.

Eighteen questionnaires were distributed to aged-care workers at RSL Care Tantula Rise because this was the total number of care workers who used the electronic documentation system and were available for the survey. 
Fifteen copies were returned, a response rate of $83.3 \%$. Twenty-three copies of the questionnaires were distributed at RSL Care Centaur. Five reminder calls were made to the facility management to follow up. Finally ten copies were returned, response rate is $43.5 \%$.

Only five out of the 118 question items used to test the 19 areas investigated were not comparable between the two sites. Therefore, Man-Whitney U test was conducted to identify if the difference in results for the remaining 113 question items was significant between the two sites. The $95 \%$ confidence interval was assumed for differences between groups of samples. Quantitative data analysis was conducted using statistical software SPSS.

\section{A. The electronic nursing documentation system}

$R S L$ Care has attempted to introduce an electronic nursing documentation software system at their new Tantula Rise Retirement Community located at Alexandra Headland, Sunshine Coast. The system is not limited to nurse managers, but is extended to personnel in all areas and at all levels of care.

The infrastructure for the electronic documentation system includes a wireless network, thirteen tablet PCs, several desktop computers and a commercial electronic documentation software. The system is not limited to higher-level users such as nurse managers, but is extended to personnel in all areas and at all levels of care. The vendor Company has custom-developed the software according to $R S L$ Care's residential aged care information management practice and is required to be designed to fully satisfy $R S L$ Care's residential care documentation requirements and workflow.

\section{Results and Implications}

The demographic profile of the care workers in the two nursing homes was similar. Although only started to work in RSL Care Tatula Rise since the nursing home was opened in August 2005, all of the care workers participated in the survey had previous aged care work experience, the majority of them with other aged care service providers. Therefore, they were familiar with traditional paper-based documentation practice.

Interestingly, only responses to 18 question items $(15.9 \%$ of the 113 question items) were significantly different between the electronic and paper-based documentation site, whereas the care workers' answers to the remaining 95 question items $(84.1 \%)$ at both sites were similar. This suggests that the two nursing homes shared similar characteristics. Although only being established for five weeks at the time of the survey, the electronic documentation site appears to match its paper-based counterpart in the areas investigated.
At this early stage of electronic documentation system introduction, the above results indicate that the impact of the electronic documentation software system on the delivery of residential care at the electronic documentation site is similar to that of the paper-based site.

The following sections will discuss the important findings in regard with several critical concerns of management on the introduction of clinical IT solutions: time and effort in documentation, and the care workers' acceptance and use of this new IT innovation.

\section{A. What software functions did the care workers like to use?}

The care givers were most happy with

1) 'Care alerts' that facilitates sharing of information among the members of the care team

The majority of the care workers interviewed expressed their satisfaction with the 'care alerts' function, which can be highlighted by a care worker if she believes that the information is important and requires the attention of the other team members. This function enhances the visibility of important information that should be shared in the care team. An interviewee explained "The system is set up in such a way that it won't let you go any further unless you read certain notes. So if I wrote, as far as communicating, if there was an alert or an important message that I need to let the staff know, if I write that in the computer as 'care alerts', it can't get missed. However, if it was written on the notes they would have to go back through rooms and rooms of paper to find out what I had written."

2) The classification system that enhances the care workers' awareness about the care to be delivered

As the information in the electronic documentation system is classified, it makes the care workers think differently. The facility manager explained this as "if I had one PRN medication, it will bring that classification up. So every time they $(R N s)$ bring that classification up, they are going to think why they did that. Because you have classification, you have to write down in the progress notes to make care workers think more carefully before they take action." This point was also mentioned by several other interviewees.

\section{B. Does the electronic documentation system reduce documentation time?}

At the questionnaire survey conducted five-weeks into the product trial, the aged care workers at the electronic documentation site gave significantly more positive answer in regard with their documentation system "save time", "put all the information in one place" and "increasing legibility" than that from the paper-based site $(\mathrm{p}<0.05)$. After using the system for 11-weeks, the nine care workers interviewed at the electronic documentation site all agreed that conducting nursing documentation in the electronic environment is quicker than that using traditional paper and pen. 
Where does time-saving come from? A personal care worker (PCW) explained "for writing even if I make a spelling mistake it is there straight away. I would have to rewrite and sign it, because it is a legal document. But here if there is a spelling mistake straight away it comes up 'Oh I spelt that wrong, ok'. And the spelling check goes zip. It's great". Another PCW said "You've got access to all the files at your fingertips. You're not running backwards and forwards."

Legibility of information is also an important recognised benefit, as a PCW said "well you can read it. There is no trouble reading it. With some people's handwriting you can't always read it."Although the time necessary for other procedures involved in care assessment and decision making was perceived to be the same, the formulation of nursing documentation components is expedited by the ease of access and legibility of data required for their creation. This, again, should lead to time saving.

At the time of the survey, no statistically significant difference was found between the two sites in terms of organisational culture and teamwork climate. This leads to the believe that the culture and teamwork climate between the two sites were not influenced by their different nursing documentation systems.

\section{Ease of use lead to end user acceptance}

As not all of the care workers have a good understanding of IT system, some of them were afraid to make mistakes and loosing data. These people tend to blame themselves if things go wrong, which cause them anxiety. This was addressed by an endorsed enrolled nurse "Yeah I think people get a bit put off with the fact that they think it is a big complex complicated system. I think they need to not necessarily take more time ... I think what most people are frightened of is if they make a mistake. They might break it. People get a bit frightened of things because they have to sort through all the technical issues that they were not confident about". This provides us with insight about the possible difficulties and the perceptions of the end users with low computer skills towards newly introduced IT innovation. It suggests that the end users are expecting the IT systems to be simple and easy to use. If the system needs considerable effort to learn, then its chance of acceptance by the end users is low.

The low acceptance of 'handwriting' function of pen on the tablets also suggests that technical stability and ease of use is critical for the acceptance of IT technology by aged care workers. 10-weeks into the product trial, none of the care workers had used the 'pen' to enter data into tablets. Instead, they were using keyboards to type data, despite some senior-aged care givers were very slow in typing. Some care workers explained to the author that they did not like the feeling of 'pen' touch on the screen. This is also attributed to the inaccurate handwriting recognition provided by the tablets used in the trial.

D. The positive usage experience has significantly improved the care workers' acceptance of electronic information system at the electronic documentation site

None of the care workers at the electronic documentation site agreed that "computer creates more problems than they solve in residential care", whereas $30 \%$ of the care workers at the paper-based site agreed with the statement. Only $6.7 \%$ of the respondents at the electronic documentation site slightly agreed that "the use of electronic documentation system dehumanises residential aged care", whereas $20 \%$ of the care workers at the paper-documentation site agreed with the statement. This suggests that the positive work experience with this clinical IT solution has decreased the care workers' concerns about the negative impact of the system. Instead, without any positive experience with a real electronic documentation system, the care workers at the paper-based site were still suspicious about the benefits of electronic documentation on aged care service delivery.

All of the care workers at the electronic documentation site expressed their commitment to learning (or have learned to use) the electronic documentation system and disagreed that "working with a computer makes me very nervous". By comparison, only half of the care workers at the paper-based site were committed to learning the electronic documentation system and $40 \%$ of them felt nervous about working with a computer. Finally, $53.3 \%$ of the care workers at the electronic documentation site have a lot of self-confidence on working with computers, whereas only $30 \%$ of the respondents at the paper-based site had this level of confidence.

Even without any experience with an electronic nursing documentation system, and with the fact that many of them are not confident computer users and felt threatened by the perceived uncertainty of change that would inevitably be caused by the introduction of the electronic system, $70 \%$ of the care workers at the paper-based site expressed their support for RSL Care's decision to introduce the electronic documentation system. An increased support rate (86.7\%) was recorded at the electronic documentation site. This suggests that the aged care workforce in Australia is keen to embrace clinical IT innovations.

\section{E. The importance of education and support for improving quality of nursing documentation}

The facility manager saw it as one gap with the electronic documentation system that it would not change the processes and quality of nursing documentation unless the extra support mechanisms for documentation is in place. This emphasis on education and support on nursing documentation was also highlighted by several care workers the author interviewed. 
After 10-week's interaction with the software, these care workers said that they did not need any further training on how to use the electronic documentation software. However, many of them still saw it as a challenge for them to distinguish what to document and how to document in order to comply with RSL Care's documentation standard.

\section{Conclusion}

Data collected 5 - 11 weeks into the electronic documentation trial at RSL Care Tantula Rise indicates that this clinical IT introduction into residential aged care was a great success in terms of acceptance by all types and levels of occupations involved in care service delivery. This success has a profound impact on the introduction of information technology in the residential aged care sector, because up until now, despite the substantial national effort placed on the introduction of electronic health records into healthcare in Australia, the uptake of clinical IT innovation in residential aged care has been very limited. There remains a pervasive scepticism about the capacity of 40-60 year-old care workers to use computers. Also past research has found that nurses' confidence with computers is in inverse proportion to their years of nursing practice. The enthusiastic and successful adoption of the electronic documentation system at RSL Care Tantula Rise, however, proves that the 40-60 year-old care workers are both willing and able to adopt modern, computer-based nursing documentation practice.

\section{A. Limitations of the study}

The care workers' perceptions of and attitudes towards the implemented electronic documentation system may change as time goes on, but this study is limited to the results measured at 5 and 11 weeks after the implementation of the electronic documentation system. Although the response rate at the electronic site was reasonable, the total sample size was small. Both nursing homes belong to the same aged-care management group; consequently, this may limit the generalisability of the research findings. Also, the sensitivity of non-parametric data analysis is not as strong as parametric test. Misinterpretation and bias may arise from analysing self-reported questionnaire data. Therefore, the interpretation of the results in this paper should be treated with caution. However, the results are relevant within the sites where the study was conducted.

\section{Acknowledgement}

The Chief Executive Officer of RSL Care Ross Smith provided valuable input to the aim, planning and conduct of the project. Members of the Project Steering Committee: Deborah Oxlade, Cheryl Richardson, Matthew Maw, Steve Powell, Susan DeVries, Susan Lane and Michelle Coman facilitated the whole process of investigation. This includes survey instrument development, validation and approval, facilitating the conduct of field survey and commenting on interim report. Without the participation of care staff at the two nursing homes, this study would have been impossible. Dr. Madeleine Strong Cincotta has contributed to editing and proof-reading the manuscript.

\section{References}

[1] Crofton and Witney (2004) Nursing documentation in aged care: a guide to practice. Melbourne: Ausmed Publications Pty Ltd.

[2] Pelletier, D., C. Duffield, et al. (2002). "The complexities of documenting clinical information in long-term care settings in Australia." Journal of Gerontological Nursing 28(5).

[3] Fitzroy, N. and S. Moseby (2002). "The stress of aged care documentation." Australian Nursing Journal 9(10).

[4] Moseby S. The stress of aged-care documentation. Australian Nursing Journal 2002; 9: 27.

[5] Richardson S, Martin B. The care of older Australians: a picture of the residential aged-care workforce. Adelaide: The National Institute of Labour Studies, Flinders University, 2004.

http://www.health.gov.au/internet/wcms/publishing.nsf/Conte nt/ageing-workforce-workforcerep.htm-copy2 (accessed 10 Feb 2006).

[6] Oritz, E., \& Clancy, C. M. (2003). Use of information technology to improve the Quality of Health Care in the United States. Health Services Research, 38(2), 11-22.

[7] Hogan WP. Review of pricing arrangements in residential aged-care: final report. Canberra: Commonwealth of Australia, 2004.

http://www.health.gov.au/internet/wcms/publishing.nsf/Conte nt/health-investinginagedcare-report-index.htm (accessed 10 Feb 2006)

[8] Australian Department of Health and Ageing. Clinical IT in aged-care: Interim report. Canberra: Australian Department of Health and Ageing: 2003. http://www.health.gov.au/internet/wcms/publishing.nsf/e11ff a331b366c54ca2569210006982f/ageing-rescare-clinitrep.htm (accessed 10 Feb 2006).

[9] Demirjian S, Chaczko Z, Hughes J. Information technology and the residential aged-care workforce. Sydney: Faculty of Engineering, University of Technology 2004 (Unpublished report.)

[10] Stoil MJ. Technology as a solution-And a policy problem? Nursing Homes 2004; 53: 12-13.

Ping Yu is with School of Information Systems and Technology, The University of Wollongong, NSW, Australia 2522 (Tel: 0061-2-42215412; Fax: 0061-2-42214045; email: ping@uow.edu.au).

Hui $\mathrm{Yu}$ is with School of Biomedical Science, Chongqing Institute of Technology, Chongqing, P.R.China 400050 (colinhuiyu@yahoo.com).

Yi Mu is with School of Computer Science and software Engineering, The University of Wollongong, NSW, Australia 2522. (Tel: 0061-2-42215228; Fax: 0061-2-42214170; email: ymu@uow.edu.au) 